\title{
In-Situ Observation of Crystal Alignment under a Magnetic Field Using X-ray Diffraction
}

\author{
Takenori Kohama $^{1, * 1}$, Hiroki Takeuchi ${ }^{1, * 1}$, Manabu Usui ${ }^{1, * 2}$, Jun Akiyama ${ }^{1, * 3}$, \\ Mun-Gyu Sung ${ }^{1}$, Kazuhiko Iwai ${ }^{1}$ and Shigeo Asai ${ }^{2}$ \\ ${ }^{1}$ Department of Materials, Physics and Energy Engineering, Nagoya University, Nagoya 464-8603, Japan \\ ${ }^{2}$ Innovation Plaza Tokai Japan Science and Technology Agency, Nagoya 457-0063, Japan
}

\begin{abstract}
The crystal alignment behavior of bismuth particles in the presence of an imposed static magnetic field was examined in situ by X-ray diffraction. Because the c-plane of a bismuth crystal is aligned perpendicular to the direction of a magnetic field, the temporal variation in the (110) peak intensity of bismuth was measured by X-ray diffraction to determine the crystal alignment. The alignment time decreased as the magnetic field strength increased. This tendency is similar to that calculated for the relaxation time. The difference in the magnetic susceptibility between the magnetically easy and hard axes is the driving force for the crystal alignment, and aggregation of the bismuth particles decreases this driving force. The effective difference in magnetic susceptibility for aggregated bismuth particles was estimated by measuring the alignment time of the particles under magnetic fields of various strengths. The estimated effective difference in magnetic susceptibility generally increases with a decreasing magnetic field strength. Furthermore, the interference to crystal rotation caused by the interaction between the induced current and the imposed magnetic field is negligible in this study. To decrease the strength of the magnetic field required for alignment of crystals, the number of small particles should be reduced. [doi:10.2320/matertrans.MI200719]
\end{abstract}

(Received June 12, 2007; Accepted August 24, 2007; Published October 25, 2007)

Keywords: crystal alignment, X-ray diffraction, magnetic anisotropy, in-situ observation, magnetic susceptibility

\section{Introduction}

A magnetic field can have useful functions, such as crystal alignment, ${ }^{1)}$ levitation, ${ }^{2)}$ separation, ${ }^{3)}$ and flow control of an electrically conductive fluid. In particular, crystal alignment is an attractive tool for producing functional materials, because physical properties of materials, such as the electric, magnetic, thermal, and mechanical properties, can be controlled if these properties have an anisotropic nature. With the development of the technology of superconducting magnets, crystals of nonmagnetic, magnetically anisotropic materials such as metals, ${ }^{4-6)}$ ceramics, ${ }^{7,8)}$ and polymers ${ }^{9)}$ can be aligned by the imposition of a strong magnetic field. To form unidirectionally aligned crystals for a material with a magnetic anisotropy of $\chi_{\mathrm{c}}<\chi_{\mathrm{a}}$, a combined process involving the imposition of a magnetic field and the rotation of a sample has been proposed. ${ }^{9,10)}$ By using this method, a bulk form of hydroxyapatite with unidirectionally aligned crystals has been produced. ${ }^{11)}$ A theoretical analysis for the optimization of this process has been performed. ${ }^{12)}$ Furthermore, crystal alignment of a tin-lead binary alloy primary phase in a magnetically preferred direction can be controlled by changing the duration of an imposed electric current and static magnetic field during solidification. ${ }^{13)}$ For optimization of these crystal-alignment processes, the rotation behavior of crystals suspended in a solvent in a magnetic field should be clarified.

We examined the in situ crystal-alignment behavior of bismuth particles in the presence of a static magnetic field by in situ X-ray diffraction (XRD) studies.

\footnotetext{
${ }^{* 1}$ Graduate Student, Nagoya University

${ }^{* 2}$ Graduate Student, Nagoya University, Present address: IBIDEN Co., LTD, Ogaki 503-8604, Japan

${ }^{* 3}$ Graduate Student, Nagoya University, Present address: Institute for Molecular Science, Okazaki 444-8585, Japan
}

\section{Experimental Details}

The experimental apparatus is shown in Fig. 1. Bismuth particles $(2.67 \mathrm{~g})$ were mixed with liquid polydimethylsiloxane $(4 \mathrm{~mL})$ to form a slurry. The viscosity and density of the polydimethylsiloxane were $50 \mathrm{~Pa} . \mathrm{s}$ and $1000 \mathrm{~kg} / \mathrm{m}^{3}$, respectively. An acrylic vessel containing the slurry was placed in the bore of a superconducting magnet for the imposition of a vertical static magnetic field on the slurry. The size distribution of the bismuth particles used in this experiment is shown in Fig. 2. The mean diameter was $8.25 \mu \mathrm{m}$. The

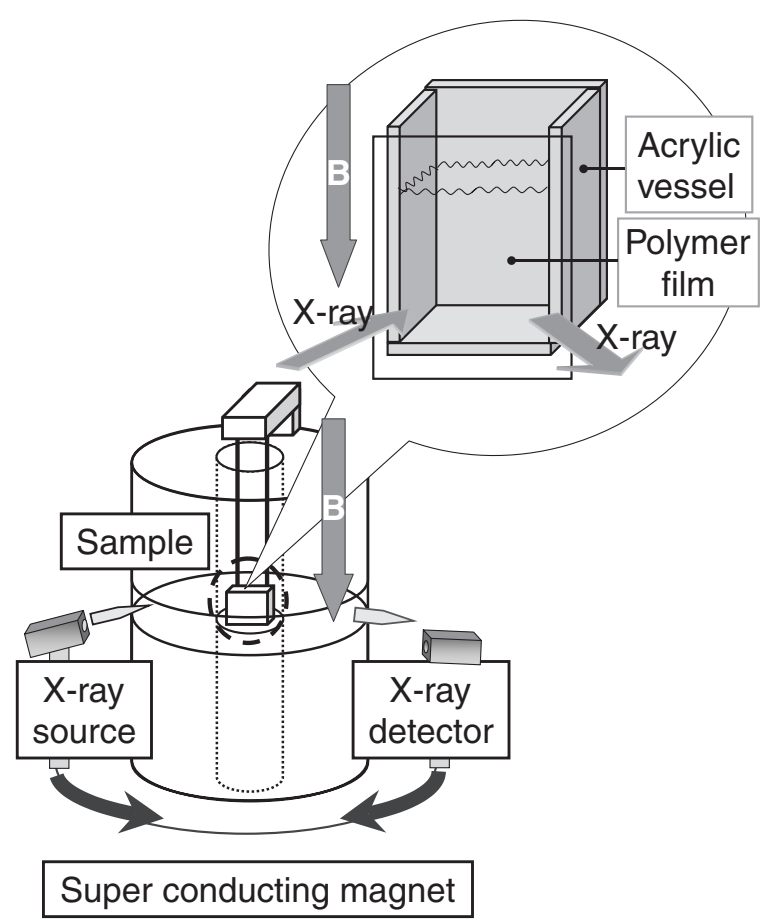

Fig. 1 Experimental apparatus. 


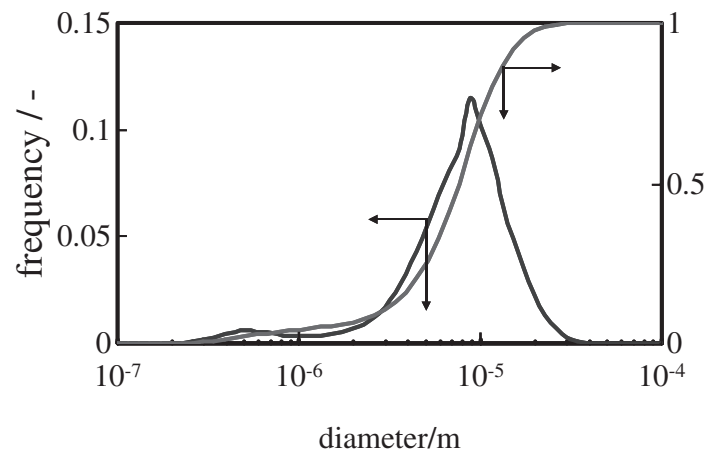

Fig. 2 Size distribution of bismuth powder.

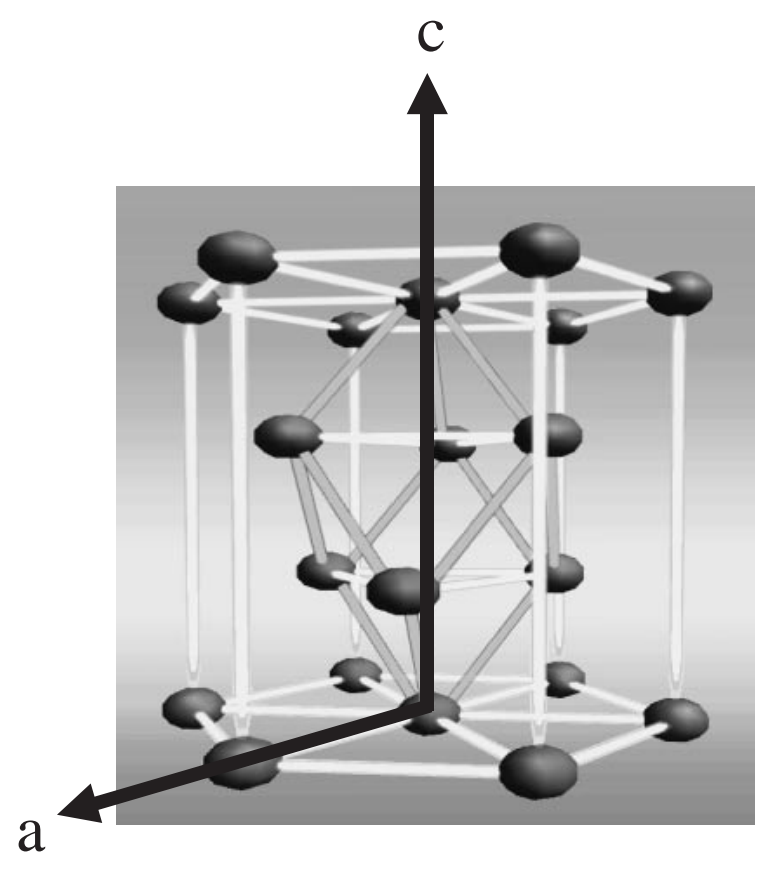

Fig. 3 Crystallographic structure of bismuth.

ratio of particles with diameters of less than $0.389 \mu \mathrm{m}, 1 \mu \mathrm{m}$, and $20 \mu \mathrm{m}$ were $1.0,4.4$, and 98 vol. $\%$, respectively.

The crystallographic structure of bismuth can be considered as a hexagonal structure, as shown in Fig. 3, and bismuth crystals show anisotropy in magnetic susceptibility. Because the magnetic susceptibility of bismuth in the direction of the a-axis is $-1.77 \times 10^{-4}$ and that in the direction of the c-axis is $-1.24 \times 10^{-4}$, the c-axis must be parallel to the magnetic field direction. This means that the peak XRD intensity in a plane parallel to the c-axis increases on imposition of a vertical magnetic field in this experiment, because the X-ray direction is in a horizontal plane, as shown in Fig. 1.

The effective difference in magnetic susceptibility of the bismuth particles used in this experiment must be small in comparison with that of a single crystal, because the bismuth particles with nonspherical shapes aggregate, as shown in Fig. 4.

The XRD results in the presence and absence of a magnetic field are shown in Fig. 5. The (110) peak is intensified by the imposed magnetic field, whereas the (104) peak is sup-

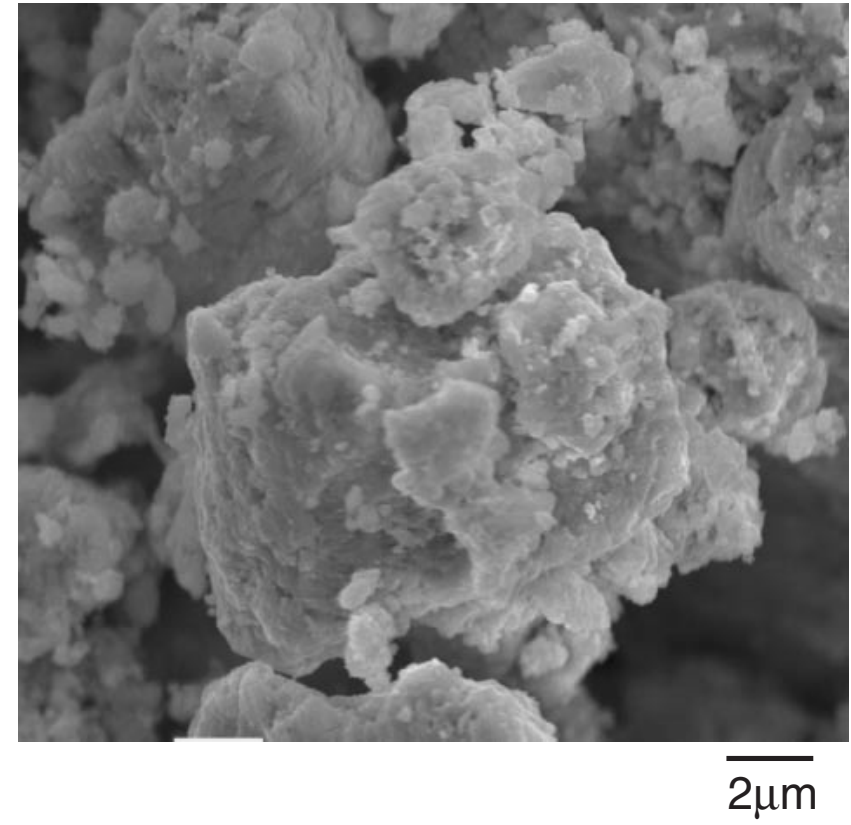

Fig. 4 SEM picture of bismuth particles.

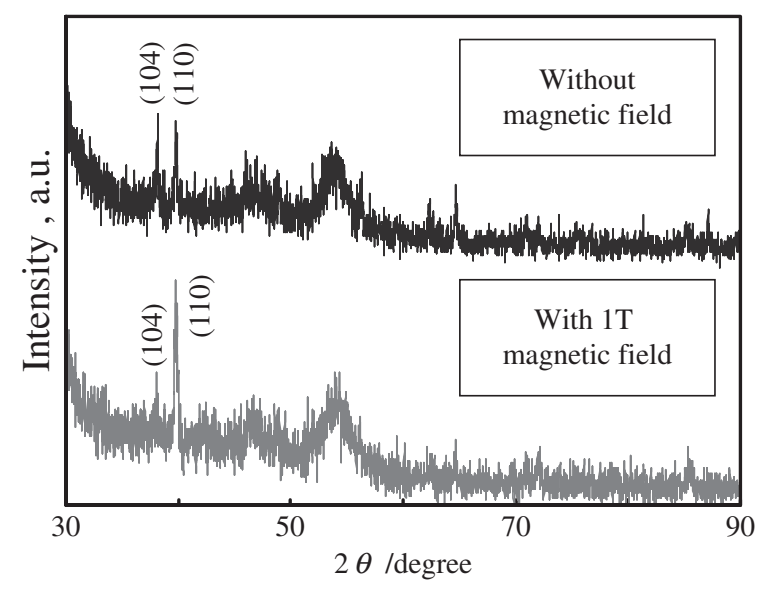

Fig. 5 XRD results with and without magnetic field.

pressed. Therefore, the (110) intensity of the bismuth crystal was chosen as an index of the crystal alignment, and its temporal variation was measured for various strengths of the magnetic field. The (110) peak angle, which was $39.6^{\circ}$ in the absence of the magnetic field, changed slightly on imposition of the field. We therefore searched for the peak angle between $39.4^{\circ}$ and $39.8^{\circ}$ in $0.01^{\circ}$ increments: this search took about $30 \mathrm{~s}$. The results measured in magnetic fields of 0,1 , and $2 \mathrm{~T}$ are shown in Fig. 6. The (110) peak intensity did not change with time, although it showed a small fluctuation in the absence of the magnetic field. On the other hand, the intensity of the (110) peak measured in a $1 \mathrm{~T}$ magnetic field increased gradually with time and it reached a plateau after a certain time. That is, the bismuth particles in the polydimethylsiloxane rotate to the magnetically preferred direction. The alignment time in this experiment was defined as the period from the insertion of the sample into the bore of the magnet to the time at which the (110) peak intensity reached $90 \%$ of its maximum value. This time is indicated in Fig. 6 by the horizontal line showing $90 \%$ of the maximum value. 


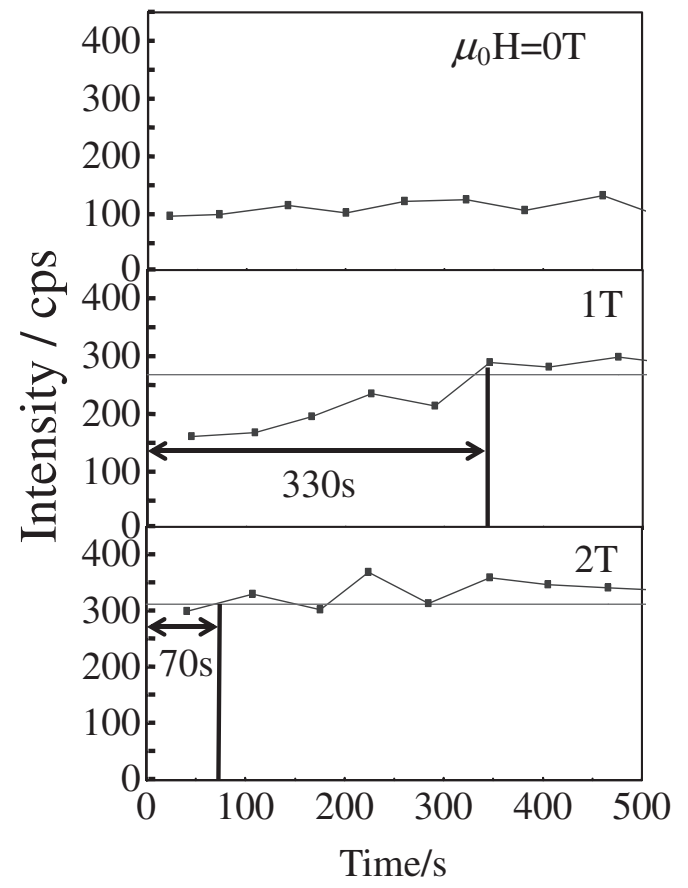

Fig. 6 Time variation of $\mathrm{Bi}(110)$ intensity.

The driving force for sedimentation of the bismuth particle is the difference in density between the bismuth particles and polydimethylsiloxane, which can be expressed as follows.

$$
F_{g}=\left(\rho_{B i}-\rho_{\text {polymer }}\right) g V_{B i}
$$

where $\mathrm{g}$ is the acceleration of gravity, $V_{B i}$ is the volume of bismuth particles, $\rho_{B i}$ is the density of bismuth, and $\rho_{\text {polymer }}$ is the density of polydimethylsiloxane, respectively.

This force is calculated as $2.31 \times 10^{-11} \mathrm{~N}$ for a particle with a mean diameter of $8.25 \mu \mathrm{m}$. Because the (110) peak intensity does not change in the absence of a magnetic field, sedimentation of the bismuth particles in this experiment can be neglected.

In the presence of the magnetic field, the magnetization force affects the motion of the bismuth particles. The following equations are used to evaluate the magnetization force.

$$
\left\{\begin{array}{l}
F_{r}=\mu_{0} \Delta \chi\left(H_{r} \frac{\partial}{\partial r} H_{r}+H_{z} \frac{\partial}{\partial z} H_{r}\right) V_{B i} \\
F_{z}=\mu_{0} \Delta \chi\left(H_{r} \frac{\partial}{\partial r} H_{z}+H_{z} \frac{\partial}{\partial z} H_{z}\right) V_{B i}
\end{array}\right.
$$

where $r$ is the radial direction, $z$ is the axial direction, $\mu_{0}$ is the magnetic permeability in a vacuum, and $\Delta \chi$ is difference in magnetic susceptibility between bismuth and polydimethylsiloxane, respectively.

The magnetization force is therefore proportional to the difference in magnetic susceptibility between the bismuth particles and the polydimethylsiloxane. The magnetic susceptibility of the polydimethylsiloxane is neglected in the calculation, because the magnetic susceptibility of polymers is usually of the order of $10^{-614)}$ which is small in comparison with that of bismuth. The calculated forces for $8.24-\mu \mathrm{m}$ diameter bismuth particles in the radial and axial directions are $\mathrm{Fr}=1.75 \times 10^{-13} \mathrm{~N}$ and $\mathrm{Fz}=9.73 \times 10^{-13} \mathrm{~N}$, respec-

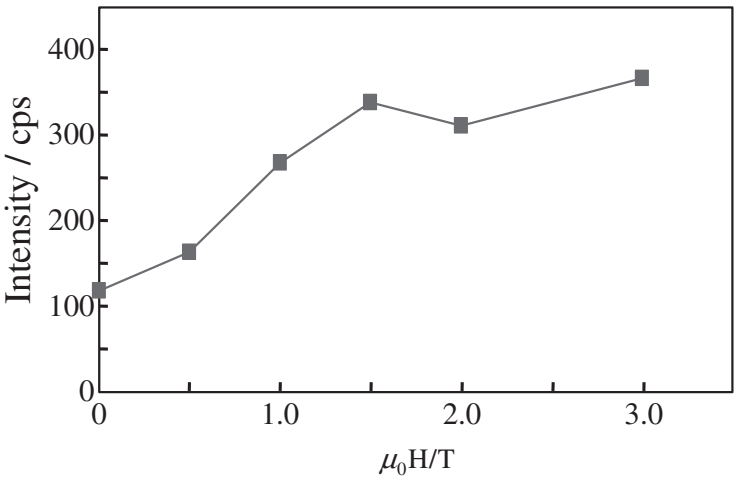

Fig. 7 Maximum Bi(110) intensity under different magnetic field strength.

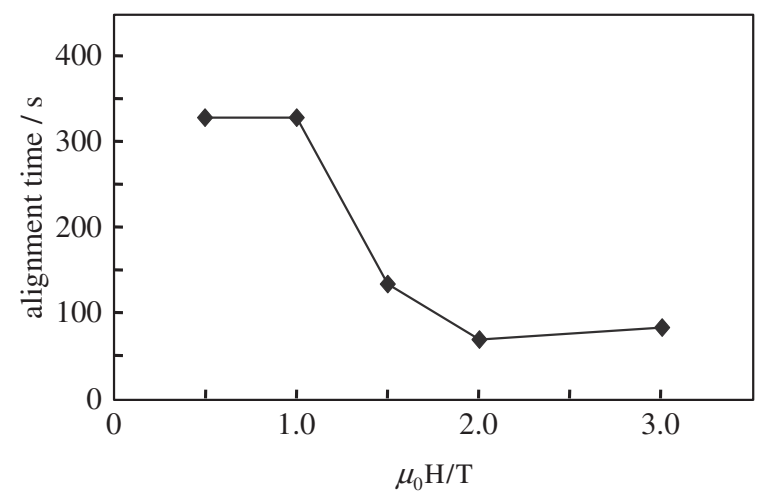

Fig. 8 Alignment time under different magnetic field strength.

tively, when the strength of the magnetic field is $3 \mathrm{~T}$. The magnetization forces can therefore be ignored in this experiment, since these forces are smaller than the force caused by the density difference.

Figure 7 shows the relation between the maximum value of the (110) peak intensity detected by XRD and the strength of the magnetic field. Because the maximum value has a positive correlation with the number of aligned particles, the number of aligned particles increases between the 0 and $1.5 \mathrm{~T}$; however, the additional effect of the magnetic field on the number of aligned particles appears to be slight at magnetic fields of more than $1.5 \mathrm{~T}$. Figure 8 shows a plot of the alignment time against the strength of the magnetic field. The alignment time decreases with increasing magnetic field strength. In particular, it decreases markedly between the $1 \mathrm{~T}$ and $2 \mathrm{~T}$. The alignment time at fields of more than $2 \mathrm{~T}$ may be weakly dependent on the strength of the magnetic field, but this is not clear from this experiment. This tendency agrees with that for the maximum magnitude, shown in Fig. 7. Therefore, almost all the bismuth particles in the vessel rotate to the magnetically preferred direction, and the rotation time is not strongly affected by the magnetic field over a certain strength of magnetic field. The critical strength in this experiment is around $2 \mathrm{~T}$.

\section{Discussion}

The law of conservation of angular momentum for a spherical single crystal suspended in a solvent with viscosity $\eta$ can be expressed as follows: 


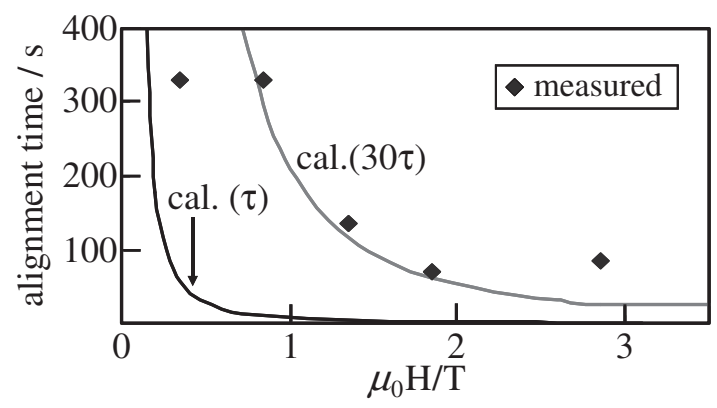

Fig. 9 Comparison of alignment time between measured one and calculated one.

$$
\begin{aligned}
& \frac{2}{5} \rho R^{5} \frac{d^{2} \theta}{d t^{2}}+8 \pi \eta R^{3} \frac{d \theta}{d t}+\frac{4}{15} \pi R^{5} \sigma \mu_{0}^{2} H^{2} \frac{d \theta}{d t} \\
& =\frac{2 \pi R^{3}}{3} \Delta \chi \mu_{0} H^{2} \sin 2 \theta
\end{aligned}
$$

where $H, R, t, \rho, \theta, \sigma$, and $\Delta \chi$ are the magnetic field strength, crystal radius, time, crystal density, the angle between the magnetically easy axis of the crystal and the magnetic field direction, the electric conductivity, and the difference in magnetic susceptibility between magnetically easy axis and magnetically hard axis, respectively.

The inertial term is dominant during the initial stages of crystal rotation. The effective time of the inertial term, $\mathrm{t}_{\mathrm{eff}}$, is estimated by equating the inertial term and the viscous term.

$$
t_{\text {eff }}=\frac{\rho R^{2}}{40 \pi \eta}
$$

The effective time is calculated to be $1 \times 10^{-10} \mathrm{~s}$ for a spherical bismuth crystal under our experimental conditions. The inertial term can therefore be neglected.

The temporal variation of the angle $\theta$ is derived from eq. (3) as follows, when the inertial term is neglected. ${ }^{15}$ )

$$
\begin{aligned}
& \tan \theta=\tan \theta_{0} \exp \left(-\frac{t}{\tau}\right) \\
& \tau=\frac{30 \eta+R^{2} \sigma \mu_{0}^{2} H^{2}}{5 \Delta \chi \mu_{0}^{2} H^{2}} \mu_{0}
\end{aligned}
$$

where $\theta_{0}$ is the initial angle between the magnetic field direction and the magnetically easy axis and $\tau$ is the relaxation time for the crystal alignment, respectively.

The relaxation time is calculated by using eq. (6) and compared with the measured alignment time. The results are shown in Fig. 9. The relation between the magnetic field strength and the measured alignment time is similar to the relation between the magnetic field strength and the calculated relaxation time, although the measured alignment time is longer than the calculated relaxation time under the same strength of magnetic field. This is because the definition of the relaxation time is different from that of the measured alignment time. Actually, the thirty times increase in the value of the relaxation time agrees well with the measured alignment time, as shown in Fig. 9. The relaxation time in the case where the electric conductivity is zero was also calculated. The calculated curve is identical to the curve in which the electric conductivity is considered, so the effect of the induced electric current on the crystal alignment can be neglected in this experiment.
Table 1 Estimated magnetic susceptibility difference of the bismuth particles.

\begin{tabular}{cc}
\hline$\mu_{0} \mathrm{H} / \mathrm{T}$ & $\Delta \chi(-)$ \\
\hline 0.5 & $2.05 \times 10^{-5}$ \\
1 & $5.13 \times 10^{-6}$ \\
1.5 & $5.57 \times 10^{-6}$ \\
2 & $6.05 \times 10^{-6}$ \\
3 & $2.21 \times 10^{-6}$ \\
\hline
\end{tabular}

The average angle between the direction of the magnetic field and the c-axis, $\theta \iota$ immediately after the imposition of the magnetic field is calculated to be one radian, by using equation (7).

$$
\theta_{i}=\frac{\int_{0}^{\pi / 2} \theta \sin \theta d \theta}{\int_{0}^{\pi / 2} \sin \theta d \theta}=1
$$

The effective magnetic susceptibility difference is then estimated from eqs. (5) and (6), by assuming that the final and initial angles are $1^{\circ}(0.017$ radians $)$ and $57.3^{\circ}$ ( 1 radian $)$, respectively. The results are listed in Table 1 . The estimated effective magnetic susceptibility difference decreases roughly with the increase in the magnetic field strength. This is because only particles with a large difference in magnetic susceptibility align in the magnetically preferred direction under a weak magnetic field, whereas particles with a small magnetic susceptibility difference also align in the magnetically preferred direction under a strong magnetic field. Actually, the effective magnetic susceptibility difference is relatively small when the (110) peak intensity, shown in Fig. 7, is relatively large, whereas the magnetic susceptibility difference is relatively large when the (110) peak intensity is relatively small.

The ratio of the magnetization energy caused by the magnetic susceptibility difference to the thermal disturbance, $X$ is indicated as follows:

$$
X=\frac{1}{2} \mu_{0} \Delta \chi H^{2} \frac{4}{3} \pi r^{3} / k_{B} T
$$

where $k_{B}$ is the Boltzmann constant and $T$ is the absolute temperature.

This ratio was calculated for various strength of the magnetic field and various crystal sizes at a temperature of $298 \mathrm{~K}$. The magnetic susceptibility differences adopted in the calculation were $5.3 \times 10^{-5}$ for a bismuth single crystal and $5.3 \times 10^{-6}$ for the bismuth particles, because the estimated effective magnetic susceptibility difference of the bismuth particles is roughly one tenth that of single crystalline bismuth in a magnetic field strength in the range $1-3 \mathrm{~T}$, as shown in Table 1. The results are shown in Fig. 10. In the case of a magnetic susceptibility difference of $5.3 \times 10^{-5}$ and a bismuth particle diameter of $8.25 \mu \mathrm{m}$, the magnetization energy is much larger than the thermal disturbance. The magnetization energy is dominant even for a bismuth particle diameter of $8.25 \mu \mathrm{m}$ and a magnetic susceptibility difference of $5.3 \times 10^{-6}$. The ratio is calculated at 268 when the particle diameter is $1 \mu \mathrm{m}$, the magnetic field strength is $1 \mathrm{~T}$, and the magnetic susceptibility difference is $5.3 \times 10^{-6}$. However, 


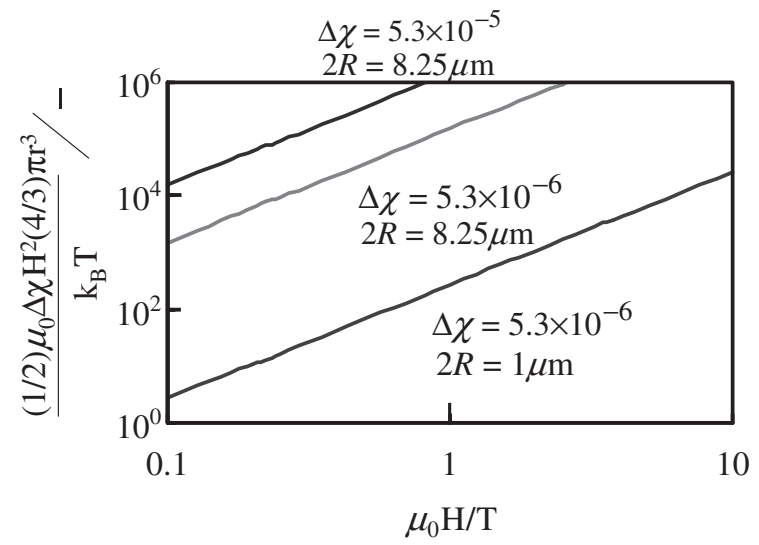

Fig. 10 Ratio of Magnetic energy difference to thermal disturbance.

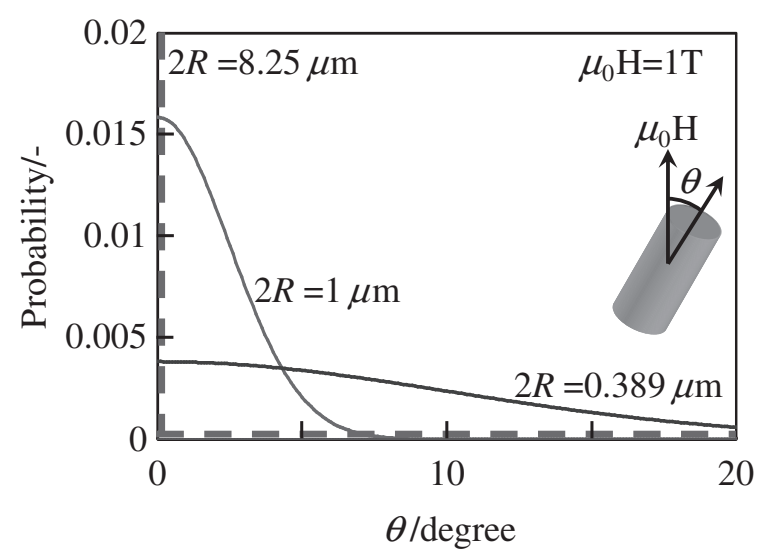

Fig. 11 Probability of angle, $\theta$ between magnetic field direction and magnetically easy axis under different crystal size.

some particles do not align in the magnetically preferred direction under our experimental conditions, as mentioned in above. Therefore, the probability of an angle $\theta$ between the magnetic field direction and the magnetically easy axis was calculated for various crystal sizes at a magnetic susceptibility difference of $5.3 \times 10^{-6} .{ }^{16)}$ The angle scattering range increases markedly as the particle size decreases, as shown in Fig. 11. Because the proportion of particles smaller than $0.389 \mu \mathrm{m}$ is $1 \%$ and of those smaller than $1 \mu \mathrm{m}$ is $4.4 \%$ in this experiment, some of these small particles are in a nonaligned state from the viewpoint of the (110) peak detection in the XRD. These nonaligned small particles reduce the maximum value of the (110) peak intensity. The angle $\theta$ concentrates by increasing the magnetic field strength under the magnetic susceptibility difference of $5.3 \times 10^{-6}$, as shown in Fig. 12 . This is the reason why the maximum value of the (110) peak intensity and the alignment time are nearly saturated at magnetic fields of more than $2 \mathrm{~T}$. These results show that the small-sized particles should be reduced to decrease the strength of the magnetic field necessary for the crystalalignment process.

\section{Conclusion}

By using XRD, we examined the alignment behavior of bismuth particles crystals in order to elucidate their rotational

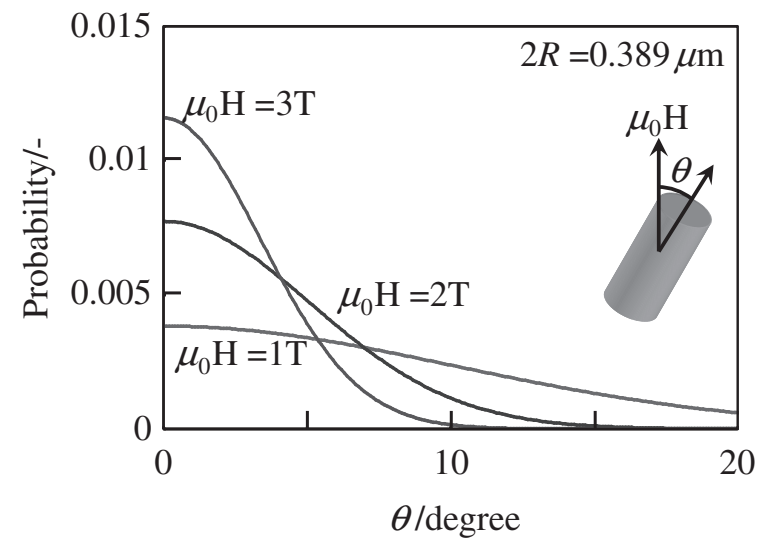

Fig. 12 Probability of angle, $\theta$ between magnetic field direction and magnetically easy axis under different magnetic field strength.

behavior when suspended in a solvent in a static magnetic field. The followings are the main results that we obtained.

\# The effective difference in magnetic susceptibility of aggregated particles can be estimated by measuring the alignment time of the particles in a magnetic field.

\# The difference in magnetic susceptibility depends on the magnetic field strength.

\# The small-sized particles should be reduced to decrease the magnetic field strength necessary for the crystalalignment process.

\section{Acknowledgement}

This research was partially supported by Iron and Steel Institute of Japan and JSPS Asian Core Program "Construction of the World Center on Electromagnetic Processing of Materials".

\section{REFERENCES}

1) S. Li, K. Sassa, K. Iwai and S. Asai: Mater. Trans. 45 (2004) 31323129.

2) K. Iwai and S. Asai: Modeling and Simulation in Materials Science and Engineering 3 (1995) 473-484.

3) Y. Tanaka, K. Sassa, K. Iwai and S. Asai: Tetsu to Hagane 81 (1995) 1120-1125.

4) H. Morikawa, K. Sassa and S. Asai: Mater. Trans. JIM 39 (1998) 814818.

5) M. Tahashi, M. Ishihara, K. Sassa and S. Asai: Mater. Trans. 44 (2003) 285-289.

6) M. Usui, K. Iwai and S. Asai: ISIJ Int. 46 (2006) 859-863.

7) T. Suzuki, Y. Sakka and K. Kitazawa: J. Cera. Soc. JP. 109 (2001) 886890.

8) Y. Sakka and S. Suzuki: J. Cera. Soc. JP. 113 (2005) 26-36.

9) T. Kimura: Polymer Journal. 35 (2003) 823-843.

10) J. Akiyama, M. Hashimoto, H. Takadama, F. Nagata, Y. Yokogawa, K. Sassa, K. Iwai and S. Asai: Mater. Trans. 46 (2005) 203-206.

11) J. Akiyama, M. Hashimoto, H. Takadama, F. Nagata, Y. Yokogawa, K. Sassa, K. Iwai and S. Asai: Mater. Trans. 46 (2005) 2514-2517.

12) J. Akiyama, H. Asano, K. Iwai and S. Asai: J. Japan Inst. Metals 71 (2007) 108-112.

13) M. Usui, K. Iwai and S. Asai: ISIJ Int. 46 (2006) 859-863.

14) M. Yamato: Kobunshi Ronbunshu 61 (2004) 433-441.

15) T. Sugiyama, M. Tahashi, K. Sassa and S. Asai: ISIJ Int. 43 (2003) 855-861.

16) K. Kitazawa: new magneto-science, (Industrial Publishing \& Consulting, Inc., Tokyo, 2002). 